\title{
Greedy Algorithm Implementation in Huffman Coding Theory
}

\author{
Author: Sunmin Lee \\ Affiliation: Seoul International School \\ E-mail: sallysmin03@gmail.com \\ <DOI:10.26821/IJSHRE.8.9.2020.8905 >
}

\begin{abstract}
All aspects of modern society depend heavily on data collection and transmission. As society grows more dependent on data, the ability to store and transmit it efficiently has become more important than ever before. The Huffman coding theory has been one of the best coding methods for data compression without loss of information. It relies heavily on a technique called a greedy algorithm, a process that "greedily", tries to find an optimal solution global solution by solving for each optimal local choice for each step of a problem. Although there is a disadvantage that it fails to consider the problem as a whole, it is definitely useful in finding the benefits locally in multi-step problems. This paper explores the algorithm in general, both the advantages and disadvantages of Huffman coding, how the Huffman coding theory uses the greedy algorithm, and ways to improve those conditions with alternatives. From details to major components, this paper will introduce Huffman coding's application and complexity as well as its variations.
\end{abstract}

\section{INTRODUCTION}

One of the major processes in data collection is translating gathered information into codes and transmitting them into various media; code can simply be thought of as a tool for communication, just like any language. However, digital technology is not a local system. It is a global one. Moreover, since no operation can be entirely independent from others, data should be interconnectable; codes have to be universal so they can serve as a bridge between each part of society. Therefore, digital technology chose numbers (binary), a universal language, in order to create the most efficient communication.
In the late 1900s and early 2000s, creating the code itself was a major challenge. However, now that the basic platform has been established, efficiency that can be achieved through data compression has become the most valuable quality current technology deeply desires to attain. Data compression, which is used to Lefficiently store, transmit and process big data such as satellite imagery, medical data, wireless telephony and database design, is a method of encoding any information (image, text, video etc.) into a format that consumes fewer bits than the original data. [8] Data compression can be of either of the two types i.e. lossy or lossless compressions. [8] Lossy compression, a non-reversible type of compression, uses approximations and discards partial data to represent a piece of the original information. With its algorithm, mostly a discrete cosine transform (DCT), this method delivers a high compression ratio (100:1), producing smaller files than any lossless method. Despite its benefits, this compression can deteriorate the quality of an image or an audio. On the other hand, in lossless compression method, no information is lost. It works by identifying and eliminating statistical redundancy to reduce the file size, and there are a number of different methods for lossless data compression such as LempelZiv, Deflate, Grammar-based codes, and Huffman coding. Lossless compression uses different probabilistic methods coupled with other algorithms, such as arithmetic coding, Run Length Encoding (RLE), Wavelets, and Fractal compression. [17]

Huffman coding is a data compression technique that uses variable-length encoding to produce a piece of data that uses a lesser number of bits to represent the same amount of data [9]. A variable number of bits are assigned to a character based on the frequency of its existence in the given text, and by generating a binary 
Volume 8 Issue 9 September 2020

tree of nodes, data is compressed. These nodes can be either an internal node or a leaf node. The internal nodes carry information about the frequency of the existence of a character, and each node is linked to two child nodes. The leaf nodes are connected via a path known as prefix code. The binary tree of the Huffman algorithm has $x$ leaf nodes and $\overline{x-1}$ internal nodes.

Huffman coding has several real-world applications such as ZIP compression, Brotli compression by Google, JPEG, MP3, MPEG-2 etc. The compressed files are stored in lesser space, can be transmitted using lesser bandwidth, and the data can be processed without any loss of information. [22]

Greedy algorithms find the overall, or globally, the optimal solution for some optimization problems, but may discover less-than-optimal solutions for some other problems. Greedy algorithms are designed to ascertain the best possible moves while executing a command. Some similar algorithms are considered greedy such as Kruskal's Minimum Spanning Tree and Dijkstra's Shortest Path First. However, both of these algorithms do not consider all possibilities and can be applied to certain problems only. One of the problems in greedy algorithms is the difficulty in figuring out the presence of other optimal solutions. Therefore, one should be skeptical of greedy algorithm solutions until it is confirmed that the greedy algorithm is appropriate for such a problem. Incorporating greedy algorithms, which can help choose the best path, into Huffman coding helps reduce the redundancy of the code. [18]

The objective of this paper is to provide insight into greedy algorithms and their potential application in Huffman coding. Overall, the paper introduces the core concepts of greedy algorithms from literature and additionally discusses coding theory and its applications in its second section. The third section provides an in-depth understanding of the Huffman algorithm; the complexity of the Huffman algorithm will be evaluated to have an idea of the algorithm execution. Lastly, variations of the algorithm based on recent publications will be presented.

\section{FORMAL DESCRIPTION OF THE GREEDY ALGORITHM}

A greedy algorithm is a direct way of simply making best choices at a local level to achieve a solution. A greedy algorithm is favored for certain problems, such as compressing data using Huffman encoding or finding the shortest path from the graph in Dijkstra's algorithm. [11] However, there are some problems in which the greedy algorithm fails to work. One such example is shown in Figure 1 below: a problem aimed to find the largest sum using a greedy algorithm. Since the optimal solution for the entire problem is neglected and every decision made is based on information available at that particular step, greedy algorithms fail to find the largest sum.

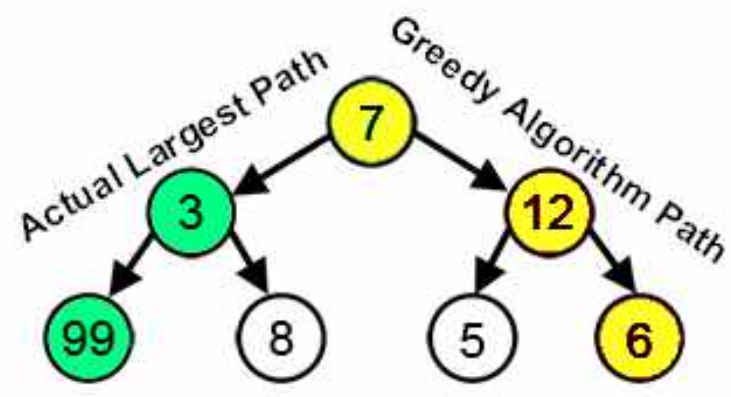

Fig 1: Greedy algorithm working to find the path for the largest sum

As it is shown in Figure 1, greedy algorithms cannot be applied to every problem. To find an optimal solution using the greedy algorithm, the following two

conditions must be met: [20]

a. An overall or global optimal solution can be reached by choosing optimal choice at every step.

b. The problem must have an optimal substructure if an optimal solution for the whole problem contains the optimal solution to the sub-problems.

Although a greedy algorithm is not applicable to every single problem, it is suitable enough for a range of different problems. Therefore, various algorithms, which are considered as applications of a greedy algorithm have been developed. Some examples include the job scheduling problem, the traveling salesman problem, the knapsack problem, Prim's minimal spanning tree algorithm, and graph-map coloring.

\subsection{Elements of a Greedy Algorithm}

There are five elements of a greedy algorithm as follows: [3]

a. Candidate set: It is the set of values for which the optimal solution is created.

b. Selection function: It is the function which chooses the best candidate that will be added next in the solution. 
Volume 8 Issue 9 September 2020

c. Feasibility function: this function determines whether a candidate can be employed to contribute to the solution.

d. Objective function: This function assign values to the partial or complete solution.

e. Solution function: It identifies the complete solution and indicates or gives the output.

Some properties of the greedy algorithm include reduction of a given problem into smaller sets by making best choices one after the other. In addition, conventional greedy algorithms do not reconsider their choices.

\subsection{Workings of a Greedy Algorithm}

Greedy algorithms evaluate feasible solutions from the subsets of a finite set and is a representation of combinatorial algorithms. The objective of the greedy algorithm is to find the optimal solution by choosing the best possible move in each step. It can be understood if viewed in isolation, the selected choice of the greedy algorithm is optimal. The question of when and why this strategy (i.e. solving more local optimum and ignoring the global) can still reach a global optimal requires an understanding of the problem itself. [7]

Breaking down the greedy algorithm reveals its two major properties. Firstly, a greedy algorithm leads to an optimum solution locally. It then follows the induction such as interval scheduling. Secondly, the solution of the greedy algorithm is as good as any other algorithm at each step. Thirdly the greedy algorithm evaluates the structural boundaries of the problem and provides the solution within that boundary. And lastly, it transforms any optimal solution from some other algorithm without altering its optimality. [14].

This can be best understood using a real-life example. Consider a student who dropped one of his courses (such as Network Architecture) and opted for Mathematical modeling. The department of mathematics offers all of its classes on the same day every week, and all the class times are different (eg. class $x$ starts at 10:00 and ends at 10:35, and class $y$ starts at 13:00 and ends at 13:40). The student wants to register a maximum number of classes to complete the degree earlier. However, the ERP system of the University will not allow any overlapping of classes.
So how will the student decide to take a maximum number of classes without overlapping any classes?

In terms of mathematics, this problem can be written as two sets of arrays i.e. $\overline{b[1 \ldots n]}$ and $\overline{e[1 \ldots n]}$. These arrays represent the beginning and ending time of the class. Here it can be assumed that $0 \leq b[i]<r[i] \leq H$. For a certain value of $H$ (time in seconds, minutes, hours etc.), the objective is to

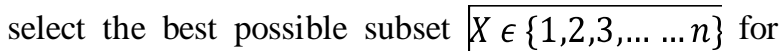

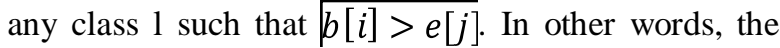
starting time of the second class must not be before the first class ends. Simplifying this problem results in a recursive solution that can be represented by rectangles. Each rectangle represents the start and end times of the class. The objective is that no rectangle should be vertically overlapping (see Figure 2).

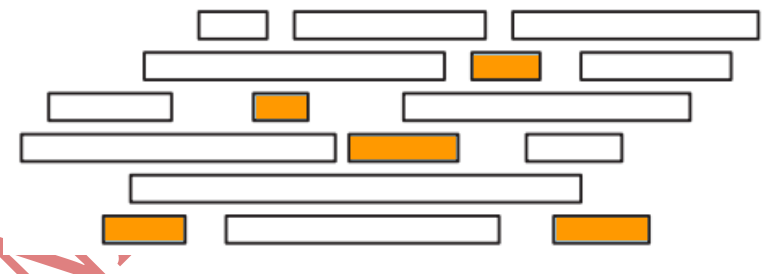

Fig 2: A conflict-free schedule with no overlapping classes

In order to take the maximum number of classes without overlapping, one would like to take the first class that ends as early as possible. This will leave the largest number of remaining classes. This hypothesis can be used to construct a very simple greedy algorithm i.e. scanning the classes based on their end time. Thus, selecting the class as soon as it is not conflicting or overlapping the other classes. The greedy algorithm will reschedule the classes as shown in Figure 3:

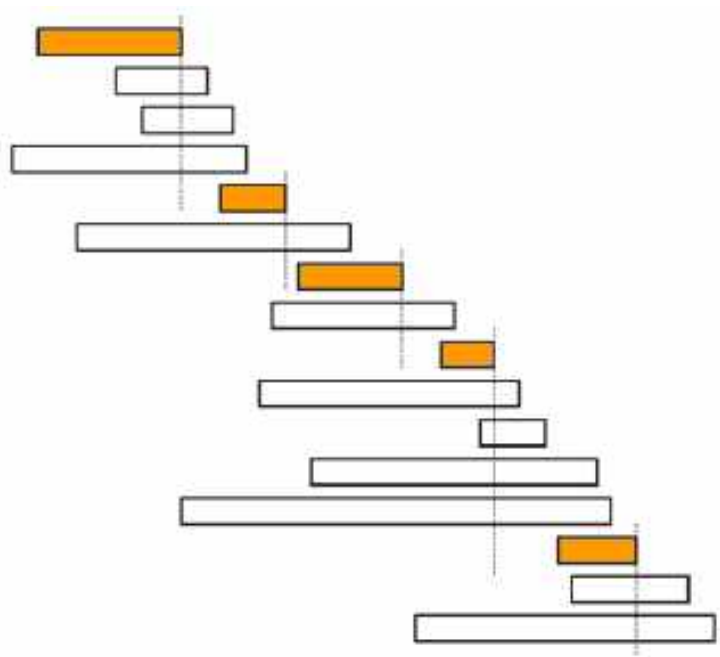


Volume 8 Issue 9 September 2020

Fig 3: Greedy Algorithm schedule based on class finish times

Another example of a working greedy algorithm is counting the desired amount of cash by choosing the least possible number of coins. Contrary to the class scheduling problem where the greedy algorithm chooses the class based on finishing time, it will select the largest denomination of the coin for this problem. For instance, if 1,5,10 and 20 coins are provided and the problem requires us to count to 36 , the greedy algorithm procedure would be as follows:

a. The first selection will be 20 (because it is the largest of 4 coins)

b. Remaining will be 16 , now the algorithm will choose 10 (because it is the largest among the 3 coins that can give the solution.

c. In the third step, it will select 5 coins and lastly coin 1 which will solve the problem. [16]

\subsection{Correctness Analysis}

Correctness analysis verifies whether the greedy algorithm has been applied correctly. The greedy correctness is proved using a form of induction. For instance, let's consider that there is an optimal solution that agrees with the first $k$ choices of the algorithm. The induction is showing that there is an optimal solution that agrees with the first $\overline{k+1}$ choices too.

There are two ways the correctness analysis can be carried out. The first method, an "experimental analysis", is a way of deploying the algorithm to the problem whose solution is already known and then checking the correctness. However, despite the fact that this approach is very simple, it is not always possible to have a solution to the problem in hand. Moreover, the testing may not be able to cover all possible instances of input data.

A second method of "formal analysis," may be more appropriate since it is based on proving an algorithm's practicality for all possible instances of input data. The advantage of this method is that it rigorously checks the algorithm for correctness. However, finding or developing proof, particularly for complex problems, might be difficult for this analysis. To solve this problem, algorithms can be broken down into subalgorithms; the proofs are developed for these simpler algorithms and then analyzed. There are three major steps involved in the formal analysis of the correctness of an algorithm as follows:

a. Identification of properties of the input data

b. Finding those properties that must be satisfied by the output data of an algorithm

c. Providing the proof that identified properties of input data and the path of the solution will produce the same outputs as produced by the greedy algorithm.

The greedy algorithm for the problem of selecting an optimal class schedule in section 2.2 can be subjected to correctness analysis. Here the purpose of the proof is not to claim that the schedule provided by a greedy algorithm is the only optimum solution. There can be other schedules that are optimum. Here the purpose of correctness analysis of the greedy algorithm is that it has produced at least one of the optimal schedules. Below is the postulate for proving this analogy.

Lemma 1: At least one optimal conflict-free class arrangement includes the class that ends first.

Let $W$ be the class that ends first. Consider a maximal conflict-free arrangement $A$ that does not include $W$ and let $p$ be the first class that finishes in $A$. Because $W$ ends before $p, W$ cannot conflict with some other class in the set $\overline{A\{p\}}$. Hence it is evident that the schedule $A^{\prime}=A U\{W\} \backslash\{p\}$ is also conflict-free. Both $A$ and $A$, have the same size as the solution is maximal.

\subsection{Computational Complexity}

While the correctness analysis of an algorithm determines whether the solution of the problem produced by the greedy algorithm is the same as the desired output, the computational complexity determines the efficiency. Computational complexity is a factor determined by considering the time required to reach the correct solution. It also includes the memory consumed by the algorithm and is a function of the size of an input instance. Therefore, the greedy complexity (run time of greedy algorithm) of maximum classes without overlapping can be found by determining the difficulty in arranging the classes at each step.

The computational complexity of greedy algorithms can be further understood by comparing Prim's algorithm to Kruskal's. Kruskal's algorithm solves the problem of the minimum spanning tree in $\overline{O\left(n^{2}+m \log n\right)}$. Where $m=|E|$ and $n=|V|$. Prim's 
Volume 8 Issue 9 September 2020

algorithm does not start with $n$ trees, which consist of single vertices and connecting these $\mathrm{n}$ trees to generate a single larger tree. Prim's algorithm approaches the problem by selecting an arbitrary vertex and generating the tree by adding a greedy edge (edge of smallest weight not yet connected from trees to vertices). Suppose a graph with weight function $H=(W, F, \varepsilon)$. We need to make a priority queue $R$ and sum up all vertices $w$ to $R$ at an infinite distance. Next is to arbitrarily select the root $s \in W$. Reduce the key of $s$ in $R$ to zero. When $\mathrm{R}$ is not empty set

$v \leftarrow \operatorname{DeleteMin}(Q)$ and for all of the edges $(v, w) \in F$, reduce the key of $w$ in $R$ to $\min (k e y R, v)$, $x(v, w)) . a$. The iterations made by Prim's algorithm are shown in Figure 4.

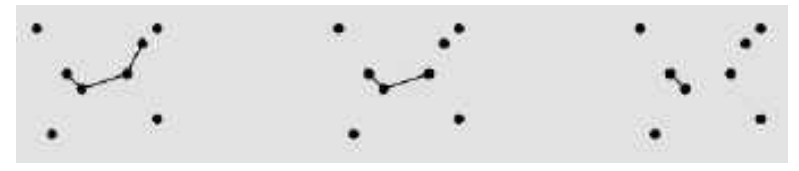

Fig 4: First few iterations of Prim's algorithm

The algorithm worked by considering $m$ inserts to $S, m$ min-deletions and $n$ decrease-keys called the implementation of the binary heap then the operations can be written as $O(\log m)$. The total complexity will be $O(m \log m+m \log n)=O(n \log m)$, which is lesser than Kruskal's algorithm.

\section{INTRODUCTION TO CODING THEORY}

Coding theory deals with the study of the properties of codes and their applications. Codes can be used for error detection, compression, data transmission, cryptography and data storage. Furthermore, they can be applied to problems such as redundancy and the detection of errors in the transmitted. There are four classes of coding i.e. line coding, source coding (data compression), channel coding (error control) and cryptographic coding. The objective of data compression coding is to remove redundancy from the data to provide more space on a storage device and transmit it efficiently over the network. The most common example of source coding is ZIP data compression, which makes the size of the file smaller. The other popular formats are RAR and 7Zip.

Source coding uses variable length code to reduce the number of characters in the given data set to a minimum. Additionally, it uses a particular code that is chosen to match the source and is independent of the path of the message. Mathematically it can be expressed as follows:

A random variable represents the data such that $Y: \varphi \rightarrow C$, where $\overline{c \in C}$ is present with a probability of $\overline{P[C=c]}$. The words represent data, where words consist of alphabets $\Sigma$.

The code represents the function such that:

$$
V: C \rightarrow \Sigma+
$$

And empty words are not part of alphabet.

$\sqrt{V(c)}$ is the coding introduced to represent $c$. The length of the representative code will be:

$$
l(V(c))
$$

Here, the possible length of code is given as:

$$
l(X)=\sum_{c \in C}(V(c)) P[C=c]
$$

The operation of joining back the multiple strings can be executed using:

$$
V\left(c_{1}, \ldots \ldots c_{n}\right)=V\left(c_{1}\right) V\left(c_{2}\right) \ldots . V\left(c_{n}\right)
$$

The string that has no values is itself a code. Therefore:

$$
V(e)=\epsilon
$$

The changes in conditions of a source, also known as entropy, is the measure of its information. Source codes reduce the redundancy of data present in the source. The result is that fewer bits are required to represent a larger amount of data. The limitation of source coding is that it is very difficult to ensure the selected shortest codewords are representing the most probable source of characters. Error correction is also necessary since through data compression, file sizes decrease and a minute loss in data during transmission will halt the regeneration process of that file.

\section{HUFFMAN CODING THEORY}

Huffman coding theory is a prefix-free coding aiming to reduce redundancy [19]. Often used for storage and efficient digital communication, Huffman coding theory is a common but important algorithm. To provide an example about its usage, consider a data file with 1 million characters. The cost to transmit or store this file is proportional to the number of bits required. How can we find the best way to store or transmit this file? For this problem, let's say there are six different symbols $(\mathrm{H})$, with the number of repetitions in the file as following: 
Volume 8 Issue 9 September 2020

Table 1. Frequency table for six symbols, A-F

\begin{tabular}{|l|l|l|l|l|l|l|}
\hline Symbols & A & B & C & D & E & F \\
\hline Frequency & 13 & 45 & 16 & 12 & 5 & 9 \\
\hline
\end{tabular}

The objective is to achieve maximum compression, so if fixed-length codes are used, only 3 bits are required to represent the 6 symbols. One such set can be represented as:

Table 2. A Normal binary representation for symbols

\begin{tabular}{|l|l|l|l|l|l|l|}
\hline Symbols & A & B & C & D & E & F \\
\hline $\begin{array}{l}\text { Binary } \\
\text { Representation }\end{array}$ & 000 & 001 & 010 & 011 & 100 & 101 \\
\hline
\end{tabular}

Therefore, it can be inferred that for a file with one million characters, 3 million bits will be used to store the file. However, the question remains: is it possible to improve it further? The solution can be provided by using variable-length codes in Huffman coding. Letters with higher frequency will have smaller codes whereas longer codes will be assigned to infrequent letters. Thus, the above table can be optimized as follows:

Table 3. Optimized binary representation based on frequency

\begin{tabular}{|l|l|l|l|l|l|l|}
\hline Symbols & A & B & C & D & E & F \\
\hline $\begin{array}{l}\text { Binary } \\
\text { Representation }\end{array}$ & 101 & 0 & 111 & 100 & 1100 & 1101 \\
\hline
\end{tabular}

Table 3 above displays codes with length ranging from one bit to 4 bits. The number of bits used by this code can be calculated as follows:

$3(13)+1(45)+3(16)+3(12)+4(5)+4(9)=224$ Kbit

Thus, implementing Huffman codes improved the fixed-length code, which consumed $300 \mathrm{~K}$ bits, by $25 \%$. However, further improvement is possible by giving the compressions of 20 to $90 \%$.

The idea of Huffman coding is simple; $n$ symbols in the input character represent initial weights attached to the set of leaf nodes, one per alphabet symbol [10]. The next step is generating a new internal node by removing at least two minimum-weight nodes using the greedy process. The sum of the weight of the internal node's two components becomes its weight. The processed is repeated by adding the internal node weight pair to the original set. The process finally stops when the set contains only one node consisting of all the original source symbols. These symbols are represented by $\overline{n-1}$ and their associated weight represented as the sum of original weights is given below:

$$
m=\sum_{i=0}^{n-1} w i
$$

For $\overline{n=6}$ symbols, whose weights are $(10,6,2,1,1$, 1), the initial leaves formed by Huffman coding will be:

$$
(0,10),(1,6),(2,3),(3,1),(4,1),(5,1)
$$

With the tie-breaking rule again, two more of the original symbols are joined in the combination step. Then, two new nodes are created, one of which has the least weight. Finally, a single node that represents all the 6 symbols is as following:

$$
([0,[[2,3]],[4,5]]]], 21)
$$

\subsection{Tree Construction}

Since creating a binary tree (a tree with at least two leaf nodes) is essential for Huffman coding due to the close relationship between the characters and the nodes, it is important to know the three core steps of a Huffman tree algorithm [1]:

Step 1: Scanning of the message, including the frequency of every character.

Step 2: Creating a single node tree for every character in the file. Lowest frequency character is placed at the root.

Step 3: Iterative unless the forest only contains a single tree. Two nodes having least-frequency are removed, creating a tree with two least frequency nodes. They are then attached to the new combined node with a frequency equal to the sum of the two-node frequencies. This new node is then inserted into the priority queue. This step is repeated until a single tree is obtained.

Encoding the compressed file can generate Huffman trees. Figure 6 below shows the two alternative code trees for $E=(8,7,6,5,4,3)$ and a minimum 
Volume 8 Issue 9 September 2020

redundancy code $Y=(2,2,3,3,3,3)$. The alphabet symbols represent the leaves, and the numerical value symbolize the weight.

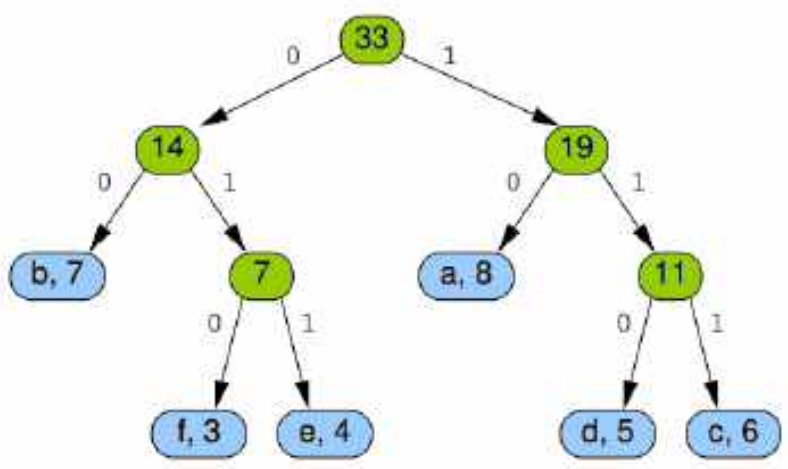

Fig 5: Huffman code trees [1]

The left tree in Figure 5 is formed by complying with the tie-breaking rule. Each pair of elements is combined, and the first node from the queue assigned to the left subtree is extracted. The second node is assigned to the subtree on the right. It's arranged based on the leaves' symbol numbers and depth.

\subsection{Greedy Elements in the Huffman Coding Algorithm}

Many greedy elements are implemented in Huffman coding. Characters are arranged based on their frequency, and with the use of a variable-length code, this algorithm assigns the shortest code to the character with the highest frequency; the shorter code takes less space. For instance, let's say there is a letter $D$ with the probability of $\overline{a(c)}$ of occurrence for every symbol $\overline{c \in V}$. The objective is to find the code $Y$ that minimizes the probable length of encoded bit-string $\overline{C(Y)}$. Utilizing the greedy algorithm will help generate the minimum length of prefix-free binary codes [21].

Furthermore, problems that may rise from variable length codes in Huffman coding can be solved by the greedy algorithm. For instance, consider 0 and 000 are codes that represent the letters "c" and "u". For certain codes, such as 00000 , decoders will have trouble decoding them. Although putting an identifying mark code is an option, it reduces efficiency. Therefore, use of a prefix code where no code is a prefix of the other one is a better solution. 0 and 000 will not be prefix codes but $(0,101,100,111,1101,1100)$. Now let's consider a letter $V$. Let the frequency of "a" in $V$ be $f(a)$. The tree for prefix code is represented by $Y$. The depth of "a" in $Y$ is represented by $\overline{d_{Y}(a)}$. The number of bits can be calculated for encoding the file as follows:

$N(Y)=\sum_{a \in V} f(a) d_{T}(P)$

This code is complex to solve for achieving the minimun possible value of $N\left(y_{8}\right.$ Here the greedy algorith $\mathrm{m}_{1}$ is used to solve for the minimum possible value of $\mathrm{N}(Y)$.

a, 8 b, 7 11 4.3 Complexity of the Huffman Coding Algorithm

The time complexity of Huffman coding 1 ean be represented as $O(m \log m)$. It contains-initial sorting as well as $m$ number of heap operations. In many cases, the time complexity is not considered when choosing the algorithm because $m$ represents the number of characters, which is very small. The complexity analysis is done only when the $m$ grows to be very large. The complexity of Huffman codes also lies in the variance of code length. This needs to be minimized because transmitting encoded data will contain an unbalanced tree with long symbols. The variance is minimized by breaking the ties between queues. This technique does not affect the optimality of Huffman coding. The technique minimizes the length as well as the variance of the largest character in the code [15].

\subsection{Variations of the Huffman Coding Algorithm}

There are several ways to find the optimal prefix codes. These methods differ due to the difference in dynamic probability selections, incorporating weights, etc. Some of the variations of Huffman coding are described below:

\subsubsection{Canonical Huffman Coding}

Using Canonical Huffman Code allows easy encoding and decoding processes. It is usually coupled with a technique known as Huffman-Shannon-Fano coding. It states that a Huffman code has the same lengths as optimal alphabetic code if the weights are represented as alphabetically ordered inputs in numerical orders [23]. This can be proved by calculating the lengths, eliminating the need of $\mathrm{Hu}$-Tucker. The minimum redundancy canonical code tree for the weights $\mathrm{W}=$ $(20,17,6,3,2,2,2,1,1,1)$ is shown in Figure 6. There are three dummy nodes on the rightmost leaf. This creates an extended sequence $W^{\prime}$ containing n'=13 leaves. 
Volume 8 Issue 9 September 2020

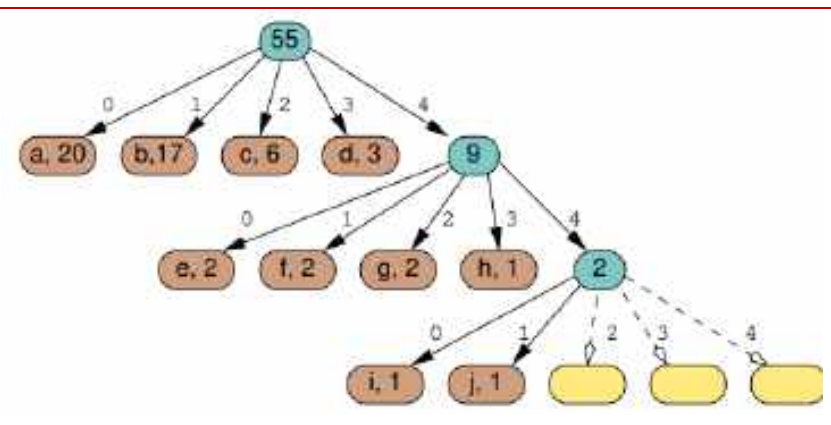

Fig 6: Canonical code tree for weights

$$
\mathrm{W}=(20,17,6,3,2,2,2,1,1,1)[23]
$$

\subsubsection{Adaptive Huffman Coding}

Adaptive Huffman coding relies on symbols from the priority list and their previous real frequencies. It is then able to determine the probabilities dynamically. However, its process might differ slightly depending on the difficulty of a problem [5]. By using "preexisting order," several adaptive algorithms can solve the problem of sorting. Manipulating the number of inversions and execution of such algorithms can provide faster results. Some studies show that the adaptive Huffman sequence for unsorted weights can be evaluated from $O\left(\left(16^{\text {Ldiff }}\right) n\right)$ time. $\mathrm{L}_{\text {diff }}$ represents the number of different codeword lengths. In another study, the adaptive Huffman code was developed where measurement of event complexity is related to the number of alterations induced by the priority input weights. It can be represented as $o(n(1+\log \propto(E))$.

\subsubsection{Length Limited Huffman Coding}

Length Limited Huffman coding's goal is to, like the original Huffman coding, reduce the weighted path length. However, the original Huffman coding puts no restriction on individual codeword length, whereas length-limited Huffman coding defines the codeword length as a given constant. "Package merge algorithm," a method incorporated into length limited Huffman coding, works very similar to the greedy algorithm. The time complexity of this coding can be written as $O(n L) ; L$ represents the predefined maximum length of the codeword [26].

\subsubsection{Huffman Template Algorithm}

In the original Huffman algorithm, weights are considered as some numerical probabilities. However, the Huffman template algorithm does not require these probabilities. It actually requires the selection of the weights from the completely arranged commutative set. This increases the option to assign a weight to different measurable quantities such as frequencies, cost, or non-numerical weights. Furthermore, it introduces different combining methods apart from simple addition, often used in circuit designs [12].

\section{CONCLUSION}

Overall, the Huffman coding algorithm has been a standard for lossless compression. However, some think the development of arithmetic coding is stepping into the limelight, with the ability to deal with highly skewed alphabets and adaptive coding. Sill, Huffman static codes have their own benefits i.e. faster execution and less memory consumption than the arithmetic coding techniques, which have two-state variables maintained

From this paper, it is evident that the greedy algorithm and Huffman coding theory are compatible with each other. Huffman coding relies on the fundamentals of the greedy approach when generating the minimum length of prefix-free binary codes, and some techniques involve direct greedy algorithm elements. In addition to present these two complementary algorithms, this paper also explained the variations of Huffman codes for different applications such as optimization of circuit design. Therefore, it can be concluded that Huffman coding is one of the most effective and efficient algorithms for data compression.

After researching Huffman coding, more questions arose regarding computational complexity. Given more time, this research project would have researched, in more depth, the different classes of complexity and how to structurally distinguish more problems. In addition, genetic algorithms and other methods of solving combinatorial optimization problems would be interesting. The fact that it was developed based on a real, biological process, natural selection, made the topic more intriguing and special. Although it was inspired from biological ideas, its current use in problems to find optimal solutions make it extremely interesting. Having similar goals with Huffman coding, genetic algorithms have an interesting connection with greedy and Huffman algorithms. Overall, there would be a number of topics to explore in these intersections. 
Volume 8 Issue 9 September 2020

\section{REFERENCES}

[1]. Habib, M. Jahirul Islam, and M. Shahidur Rahman, "Huffman based code generation algorithms: Data compression perspectives," J. Comput. Sci., 2018. vol. 14, no. 12, pp. 15991610 .

[2]. M. Raigorodskii, "Combinatorial Geometry and Coding Theory," Fundam. Informaticae, 2016. vol. 145, no. 3, pp. 359-369.

[3]. Painsky, S. Rosset, and M. Feder, "Linear Independent Component Analysis over Finite Fields: Algorithms and Bounds," IEEE Trans. Signal Process. 2018, vol. 66, no. 22, pp. 58755886.

[4]. Martel, "The expected complexity of Prim's minimum spanning tree algorithm," Inf. Process. Lett. 2002., vol. 81, no. 4, pp. 197-201,

[5]. F. Djusdek, H. Studiawan, and T. Ahmad, "Adaptive image compression using Adaptive Huffman and LZW," in Proceedings of 2016 International Conference on Information and Communication Technology and Systems, ICTS 2016, 2017, pp. 101-106.

[6]. Hoogeboom, J. W. T. Peters, R. van den Berg, and M. Welling, "Integer Discrete Flows and Lossless Compression," arXiv Prepr. arXiv1905.07376, 2019.

[7]. G. Kuchuk, V. Kharchenko, A. Kovalenko; and E. Ruchkov, "Approaches to selection of combinatorial algorithm for optimization in network traffic control of safety-critical systems," in Proceedings of 2016 IEEE East-West Design and Test Symposium, EWDTS 2016, 2016, pp. 16.

[8]. H. D. Kotha, M. Tummanapally, and V. K. Upadhyay, "Review on Lossless Compression Techniques," in Journal of Physics: Conference Series, 2019, vol. 1228, no. 1, p. 12007.

[9]. M. Pu, "Huffman coding," Fundam. Data Compression, 2006. vol. 52, no. 4, pp. 67-90.

[10]. J. Gibbons, "Coding with Asymmetric Numeral Systems," in International Conference on Mathematics of Program Construction, 2019, pp. 444-465.

[11]. L. Meng and Y. Hu, "A network coding optimization algorithm for reducing encoding nodes," in Lecture Notes of the Institute for Computer Sciences, Social-Informatics and Telecommunications Engineering, LNICST, 2018, vol. 251, pp. 265-272.
[12]. M. Senthilkumar and V. Mathivanan, "Analysis of Data Compression Techniques using Huffman Coding and Arithmetic Coding," Int. J.

Adv. Res. Comput. Sci. Softw. Eng., 2016. vol. 6, no. 5, pp. 930-936.

[13]. M. Thill, "Algebraic Techniques in Coding Theory: Entropy Vectors, Frames, and Constrained Coding", 2016 vol. 126. California Institute of Technology.

[14]. P. M. Berná, S. J. Dilworth, D. Kutzarova, T. Oikhberg, and B. Wallis, "The weighted property (A) and the greedy algorithm," J. Approx. Theory, 2019. vol. 248,

[15]. P. T. Chiou, Y. Sun, and G. S. Young, " $A$ complexity analysis of the JPEG image compression algorithm," in 2017 9th Computer Science and Electronic Engineering Conference, CEEC 2017 - Proceedings, 2017, pp. 65-70.

[16]. R. Schaback, "A Greedy Method for Solving Classes of PDE Problems," arXiv Prepr. arXiv1903.11536, 2019.

[17]. S. Alyarez-Cortes, J. Serra-Sagrista, J. Bartrina-Rapesta, and M. W. Marcellin, "Regression Wavelet Analysis for Near-Lossless Remote Sensing Data Compression," IEEE Trans. Geosci. Remote Sens., 2019. pp. 1-9,

[18]. S. J. Sarkar, N. K. Sarkar, and A. Banerjee, "A novel Huffman coding based approach to reduce the size of large data array," in Proceedings of IEEE International Conference on Circuit, Power and Computing Technologies, ICCPCT 2016, 2016, pp. 1-5.

[19]. S. Kaur and S. Singh, "Entropy Coding and Different Coding Techniques," J. Netw. Commun. Emerg. Technol. 2016. vol. 6, no. 5, pp. 4-7,.

[20]. S. Khuller, B. Raghavachari, and N. E. Young, "Greedy methods," in Handbook of Approximation Algorithms and Metaheuristics, Chapman and Hall/CRC, 2007, pp. 4-1-4-14.

[21]. S. P. Venkatesan, S. Srividhya, N. Saikumar, and G. Manikandan, "Generating strong keys using modified Huffman tree approach," in Proceedings of IEEE International Conference on Circuit, Power and Computing Technologies, ICCPCT 2016, 2016, pp. 1-4.

[22]. S. T. Klein, S. Saadia, and D. Shapira, "Forward looking huffman coding," in Lecture Notes in Computer Science (including subseries Lecture Notes in Artificial Intelligence and Lecture Notes in Bioinformatics), 2019, vol. 11532 LNCS, pp. 203-214.

[23]. S. T. Klein, T. C. Serebro, and D. Shapira, 
"Optimal skeleton Huffman trees," in Lecture

Notes in Computer Science (including subseries

Lecture Notes in Artificial Intelligence and

Lecture Notes in Bioinformatics), 2017, vol.

10508 LNCS, pp. 241-253.

[24]. W. Jiang, "Analysis and Discussion of Radar

Construction Problems with Greedy

Algorithm," in Advances in Intelligent Systems

and Computing, vol. 924, Springer, 2019, pp.

303-312.

[25]. Y. Yang, M. J. Wainwright, and M. I. Jordan,

"On the computational complexity of high-

dimensional Bayesian variable selection," Ann.

Stat., 2016. vol. 44, no. 6, pp. 2497-2532,.

[26]. Y. Yuan, Y. Zhang, Z. Liu, and X. Guan,

"Lossless coding scheme for data acquisition

under limited communication bandwidth," Digit.

Signal Process. A Rev. J., 2017. vol. 69, pp. 204-

211. 